\title{
A three dimensional modeling method for spherical open cell aluminum foams based on spherical core stratification algorithm
}

\author{
Ming-si Qi', *Wei Zhang ${ }^{2}$, Jun-yuan Wang', Guang-ming Ren ${ }^{2}$, Yi-ping Yin ${ }^{2}$, Chun-Xue Lu ${ }^{3}$ \\ 1. North University of China, Taiyuan 030051, China \\ 2. Taiyuan Heating Power Groups Co. Ltd, Taiyuan 030001, China \\ 3. Baotou North Hua'an Real Estate Co., Ltd. Baotou 014030, China
}

\begin{abstract}
Because of the complexity and irregularity of the internal structure of aluminum foam, it is difficult to establish a three-dimensional model that can accurately reflect this structure. In this study, an algorithm, named spherical core stratification algorithm, for three-dimensional modeling of spherical aluminum foam was proposed, and by using this algorithm, a three-dimensional model for sphere aluminum foam with random pores has been successfully constructed. The constructed model not only has a high similarity with the real structure of spherical open cell aluminum foam, but also can match its pore size and thickness by adjusting the size and number of holes in the random pore. In order to verify the feasibility of the modeling method, firstly, the three-dimensional model of the cylindrical spherical aluminum foam with a size of $\Phi 35 \mathrm{~mm} \times 20 \mathrm{~mm}$ and pore diameter of $5 \mathrm{~mm}$ has been generated by using the new algorithm. Secondly, taking the influence of relative density and shape function on the compressive properties of spherical open cell aluminum foams into consideration, a quasi-static constitutive model suitable for the material has been established based on the Sherwood-Frost classical compression constitutive model, which provides material parameters for quasi-static compression simulation. The comparison results show that the established constitutive equation has a good fit with the experiment, with a fitting correlation coefficient of above 0.99. Finally, the quasi-static compression simulation was carried out by ABAQUS, and the simulated nominal stress-strain curve was obtained. The simulation results indicate that the simulated stressstrain curve had the same trend with the one obtained by the quasi-static compression experiment with a small deviation.
\end{abstract}

Key words: aluminum foam; static compression; constitutive equation; 3D modeling; numerical simulation
CLC numbers: TG146.21
Document code: $\mathrm{A}$
Article ID: 1672-6421(2019)04-248-08

\begin{abstract}
A luminum foam with spherical pores is a new type of aluminum foam material as compared with irregular aluminum foam. The compressive mechanical property is the focus and challenge of research ${ }^{[1-2]}$. To date, research on the compressive mechanical properties of aluminum foam is mainly based on experiments. Simple experimental research is generalized and has poor reproducibility, and it also consumes a lot of manpower and material resources. With the rapid development of computer simulation technology ${ }^{[3-6]}$, this has become a very important method to study the compressive properties of aluminum foam through computer simulation technology. As a kind of structural metal
\end{abstract}

\section{*Wei Zhang}

Male, Master's degree. His research mainly focuses on the mechanical properties of aluminum foam and aluminum foam-polyurethane composites.

E-mail: 503655163@qq.com

Received: 2018-12-13; Accepted: 2019-06-12 material with complex internal structure, aluminum foam itself has poor reproducibility. The influence of structure on the material mechanical properties is usually summarized by statistical methods. Therefore, it is critical to identify and establish a foam metal microstructure model that can accurately reflect the relationship between cellular structure and properties of aluminum foam.

As early as 1982, Gibson and Ashby ${ }^{[7]}$ established hexahedral open-cell and closed-cell foam cells based on a single-hole model to simulate foam materials. Because it is a simple model, generating results hugely different from the real structure, it can only be used for the foam structure material with lower relative density. After having corrected and optimized this simple model, the cubic plate model was introduced, followed by the docecahedral and tetrakaidecahedral models through further model optimization. These single cell models can provide a simple and convenient method for analyzing 
the mechanical properties of materials, but their simple shape and regular arrangement are too idealized. The structure of these simple models closely approximate the real inner pore structure of the foam metal, so the data obtained from simulation are different from the real one. The Vorono ${ }^{[8-11]}$ model is a randomly arranged non periodic model. Because the structure of the model is more consistent with the internal structure of the foamed metal material, some scholars have used the Voronoi theory to establish a foamed metal material model. Most of the Voronoi models are closed cell foam metal models with irregular pore shapes, which are quite different from the structure of spherical open cell aluminum foams constructed in this study. X-ray computed tomography is a new method that can most faithfully reconstruct the structure of foamed metal materials ${ }^{[12-19]}$, and it is now used by a great number of scholars to perform $3 \mathrm{D}$ reconstruction of foam metal, due to the rapid development of this technology. However, due to the high cost of CT scanning and the relatively high professional knowledge required by researchers, the entire reconstruction process is rather complicated, leading to long reconstruction cycle and high cost.

Simulation is a common method used to verify whether the three-dimensional modeling method of aluminum foam is feasible. In order to obtain more accurate simulation results, it is necessary to establish the constitutive equation of the material to provide the necessary parameters for the simulation. The constitutive equation is a set of formulas which relate the material stress to the material strain, and it is a mathematical model reflecting the macroscopic properties of materials. The response of foam metals to plastic deformation is different from that of dense metal materials, and its constitutive equation should characterize its special response to plastic deformation ${ }^{[20]}$. At present, the constitutive model studies of foamed metal materials focuses on the quasi-static and dynamic constitutive models of foamed pure aluminum and aluminum foam alloys, and all are based on the static and dynamic test data and the existing plastic theory ${ }^{[21-23]}$. The characterization method for the constitutive model of foam metal during the plastic deformation stage is the basis of the structural design and application of foam metal. However, in practical engineering design and applications, an imperfect data set available to the material constitutive model will affect and limit the accuracy of analysis.

Compared with conventional geometry in a simplified foam aluminum model, the model built by the present algorithm can more accurately reconstruct the internal structure of spherical open cell aluminum foam. In comparison with a CT scanning $3 \mathrm{D}$ reconstruction model, the present modeling method has the benefits of a simple process, low cost and short cycle.

\section{Materials and experiment}

Sphere open-cell aluminum foam (SOAF) with an average pore size of $5 \mathrm{~mm}$ is selected as the study object. The pores have an average diameter of $1.5 \mathrm{~mm}$ for corresponding apertures. As shown in Fig. 1, a cylindrical specimen with the

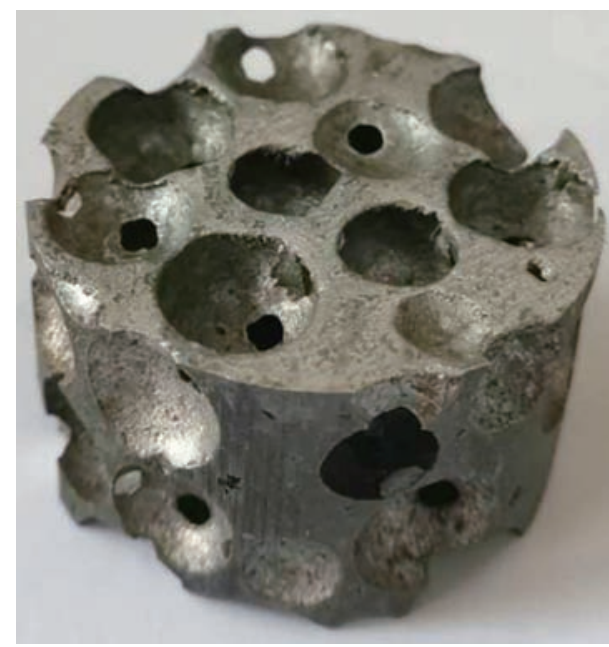

Fig. 1: Aluminum foam sample

size of $\Phi 35 \mathrm{~mm} \times 20 \mathrm{~mm}$ was cut from an aluminum foam plate using wire electrical discharge machining (WEDM), then after cleaning and drying, the aluminum foam samples were weighed with an electronic balance. The relative density $\rho_{\mathrm{r}}$ of aluminum foam is calculated from Eq. (1).

$$
\rho_{r}=\frac{\rho_{\mathrm{AF}}}{\rho_{\mathrm{S}}}
$$

where $\rho_{\mathrm{AF}}$ is the density of aluminum foam, $\rho_{\mathrm{s}}$ is the density of matrix metal aluminum and $\rho_{\mathrm{s}}$ equals $2.7 \mathrm{~g} \cdot \mathrm{cm}^{-3}$.

The quasi-static compression tests were carried out on SOAF with different relative densities by the TLS-W50000A type universal material testing machine. When the indenter loading speed was set to be $5 \mathrm{~mm} \cdot \mathrm{min}^{-1}$, the load-displacement curve was obtained through computerized data acquisition, and then the nominal stress-strain curve of the material was calculated from the load-displacement curve.

\section{3D modeling method for SOAF based on spherical core stratification algorithm}

The porosity of the open-cell aluminum foam is the ratio of the volume of all internal pores to the overall volume. This problem can be defined as follows: there are a series of hollow spheres distributed in a certain volume space, which intersect with each other and have no existing closed space. The porosity is the ratio of the volume of the pores to total volume; and the volume of pores equals to the sum of all the spheres minus the volume of intersecting regions, thus the porosity can be calculated by Eq. (2):

$$
\eta=\frac{v_{k}}{v}=\frac{\sum_{i=1}^{n} \frac{4}{3} \pi R_{i}^{3}-v^{\prime}}{v}
$$

where $\eta$ is the porosity, $v_{k}$ is the volume of pores, $v$ is the overall volume, $R_{i}$ is the radius of the $i$-th sphere, $v^{\prime}$ is the volume of intersecting regions. 
The cellular pores of the aluminum foam used in the test are spherical and have relatively regular shape, and the small round holes are open on the apertures to connect each sphere to avoid straight-through holes. Therefore, the SOAF model with nonstraight-through holes is established and shown in Fig. 2. It is a three-layer sphere superimposed structure with the purple upper layer, black middle layer and yellow lower layer, and there are six spheres distributed around each sphere in the same layer with no intersection of each other. Taking the middle layer as an example, six spheres A, B, C, D, E, and F are distributed around the center sphere $\mathrm{O}$ in the middle layer, and the centers of the six spheres are located at the points of the regular hexagon, so the inplane sphere distribution can be equivalent to the graph, formed by the base-hexagon continuously circumscribing to the adjacent hexagons. Three spheres, respectively on the upper layer and the lower layer, intersect with the spheres on the middle layer to form six-direction through-holes. When the directional angles of the three spheres on the upper layer are $\frac{\pi}{2}, \frac{5 \pi}{4}, \frac{7 \pi}{4}$, and the directional angles of the three spheres on the lower layer are $\frac{\pi}{4}$, $\frac{3 \pi}{4}, \frac{3 \pi}{2}$, the straight-through holes can be avoided, then the open-cell aluminum foam models with non-straight-through holes in six directions are formed. This algorithm is named asspherical core stratification algorithm.
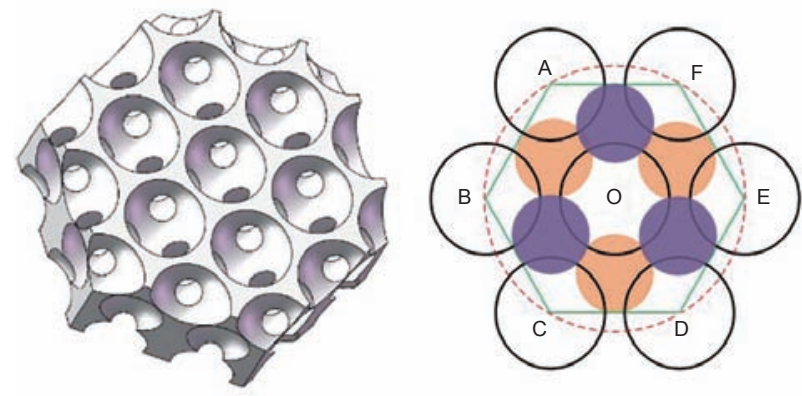

Fig. 2: SOAF model with non-straight-through holes

The algorithm flow chart for the detailed sphere open-cell foam aluminum modeling with non-straight-through holes is shown in Fig. 3. Through the algorithm, a 3D model of the internal cell of SOAF with an average pore size of $5 \mathrm{~mm}$ is established. After the solid model is created and the cell model generated by the algorithm is removed, a 3D model of open-cell aluminum foam is obtained.

The steps are as follows:

(1) Initialize to determine the diameter of the hole, the column numbers of spheres in the $X$ direction, the column numbers of spheres in the $Y$ direction, and the layers of spheres in the $Z$ direction.

(2) Calculate the side length of the hexagon in the $X O Y$ plane. Based on the idea of hexagonal circumscribing, generate the data of the sphere center of the entire first layer, that is, the vertices and centers of all hexagons in the plane.

(3) Generate data of the next layer. The current layer and the upper layer are offset and intersected with each other to form three holes. Limited to the size of aperture, the center of

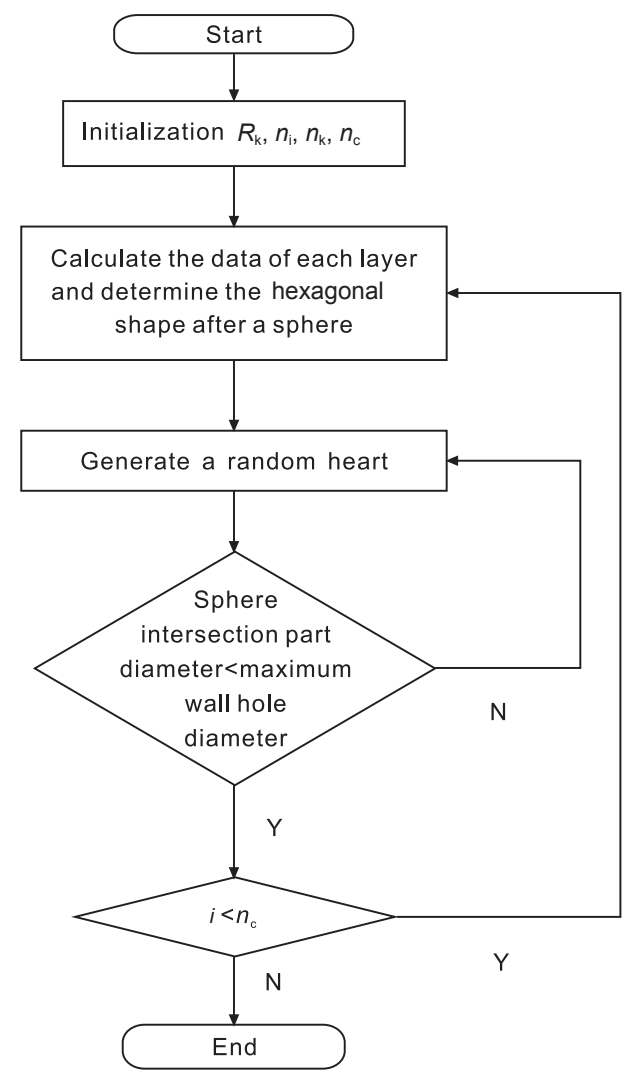

Fig. 3: Algorithm flowchart for SOAF modeling with non-straight-throughholes

the sphere is randomly generated in a small space nearby until the size of the wall hole reaches the requirement, thereby the position for one of the spheres is determined, and then the data for sphere center in this layer is generated based on the hexagon circumscription idea.

(4) Return to step (3) to solve the data of spheres on the $i$-th layer until the solving for this layer is finished.

(5) Generate the radius data of each sphere and determine the sphere at the center of each sphere, then the algorithm is finished.

The aperture size and wall size of aluminum foam materials are random values, which can be considered as normal distribution and the boundary value is interval. Taking a SOAF model with aperture size of 5-6 $\mathrm{mm}$ and and wall diameter of 1-2 $\mathrm{mm}$ as an example, the mathematic descriptions of the aperture size and wall diameter are as follows.

$$
\begin{gathered}
D_{\mathrm{k}} \sim N\left(\mu_{\mathrm{k}}, \sigma_{\mathrm{k}}{ }^{2}\right) \\
{\left[\mu_{\mathrm{k}}-3 \sigma_{\mathrm{k}}, \mu_{\mathrm{k}}+3 \sigma_{\mathrm{k}}\right]=[5,6]} \\
D_{\mathrm{b}} \sim N\left(\mu_{\mathrm{b}}, \sigma_{\mathrm{b}}{ }^{2}\right) \\
{\left[\mu_{\mathrm{b}}-3 \sigma_{\mathrm{b}}, \mu_{\mathrm{b}}+3 \sigma_{\mathrm{b}}\right]=[1,2]}
\end{gathered}
$$

where $D_{\mathrm{k}}$ and $D_{\mathrm{b}}$ are the diameters of the cell pores and the wall pores of the sphere respectively, $\mu_{\mathrm{k}}$ and $\mu_{\mathrm{b}}$ are the mean values of the cell pore diameter and the aperture diameter, $\sigma_{\mathrm{k}}$ and $\sigma_{\mathrm{b}}$ are the standard deviations of the cell pore diameter and the wall pore diameter, respectively.

Through the above-described algorithm for modeling SOAF, 

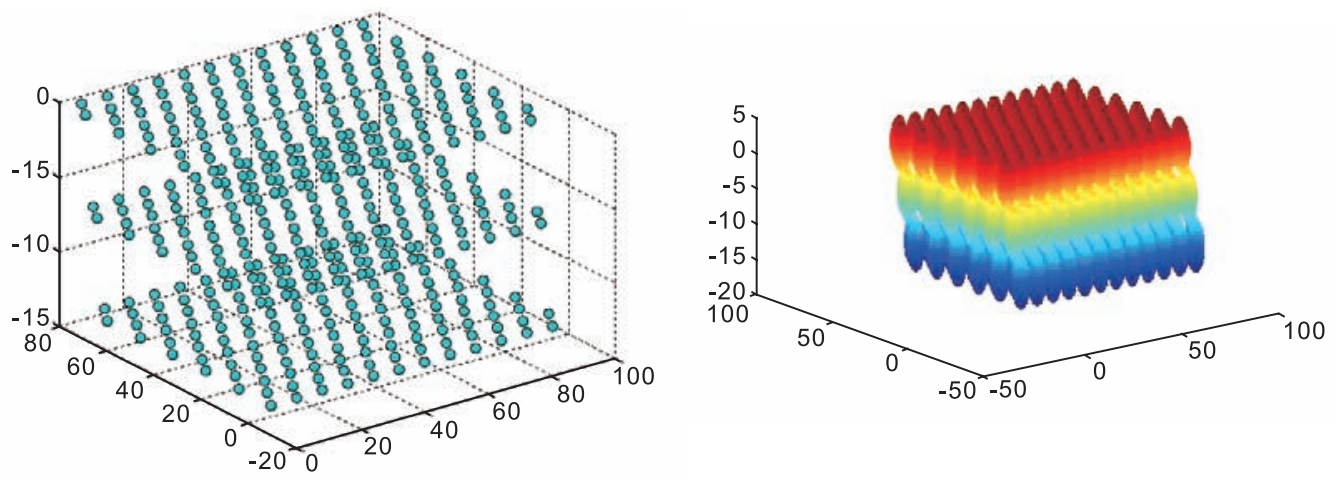

Fig. 4: Three-layer space random sphere generated by open-cell aluminum foam modeling algorithm
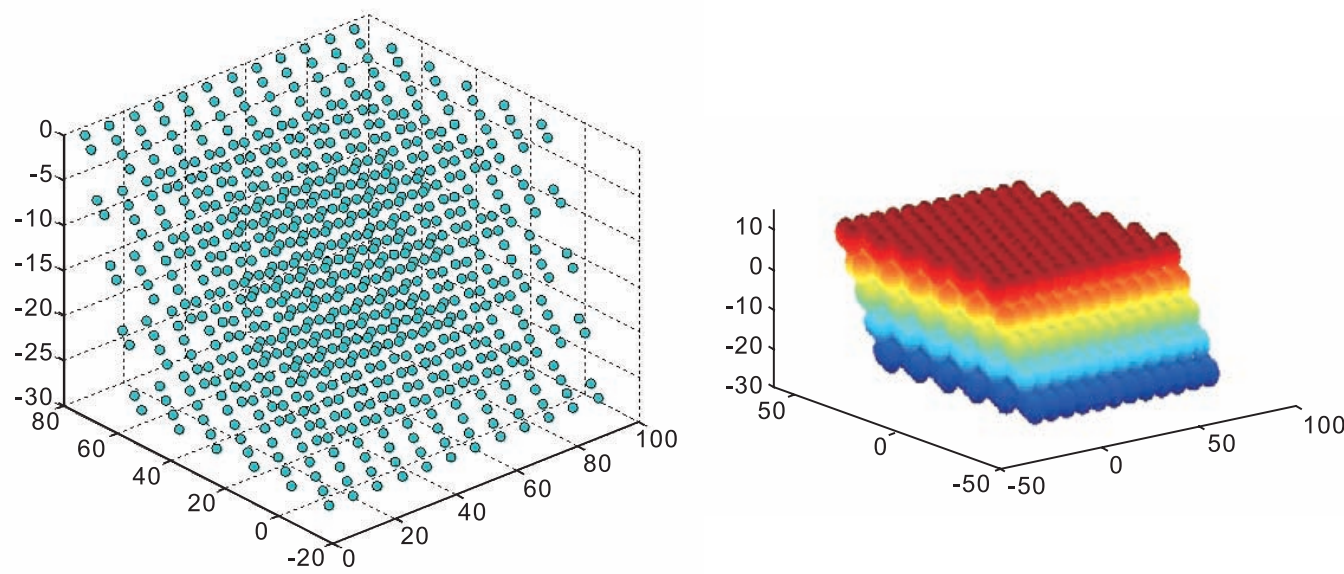

Fig. 5: Five-layer space random sphere generated by the open-cell aluminum foam modeling algorithm

the internal cell models for the three-layer and five-layer SOAF are established in MATLAB, as shown in Fig. 4 and Fig. 5. According to the open-cell foam aluminum modeling algorithm, a 3D model of $\Phi 35 \mathrm{~mm} \times 20 \mathrm{~mm}$ cylindrical SOAF is established in Solidworks using a reverse calculation method, as shown in Fig. 6.

\section{Establishment and parameter fitting of quasi-static constitutive model for SOAF}

A static compression constitutive equation is established considering only the effects of relative density and shape function, regardless of temperature and strain rate, as given in the form of Eq. (7):

$$
\sigma=G\left(\rho_{\mathrm{r}}\right) f(\varepsilon)
$$

According to the established quasi-static constitutive model, the expression forms for the relative density influencing factor $G\left(\rho_{\mathrm{r}}\right)$ and the shape function affecting factor $f(\varepsilon)$ must be determined separately.

\subsection{Influence of relative density}

In order to study the influence of relative density on the constitutive model, under the same strain rate conditions, a quasi-static uniaxial compression test was performed on aluminum foam specimens with different relative densities of $0.325,0.364,0.381$, and 0.386 respectively, and the obtained stress-strain curve is shown in Fig. 7. It is apparent that the
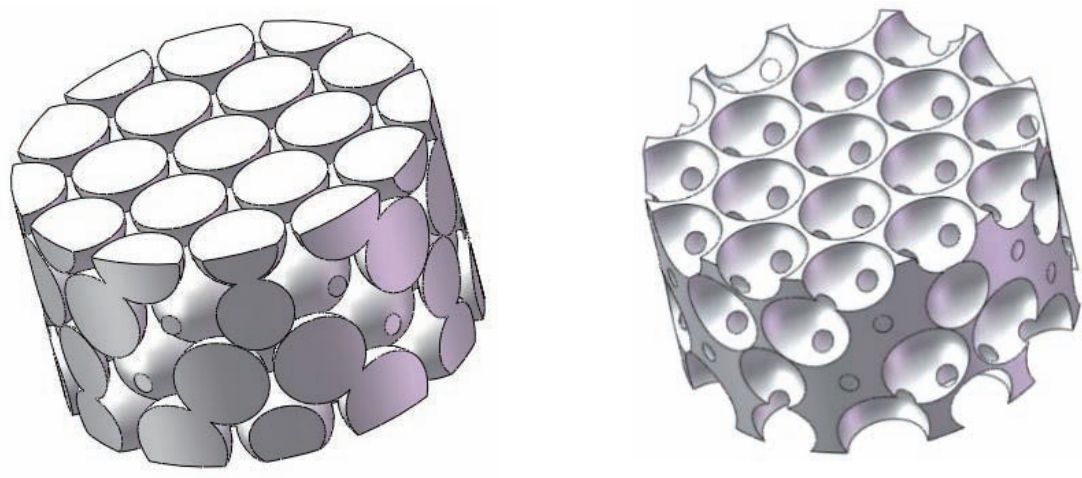

Fig. 6: Internal hole model and reverse calculation model of SOAF 


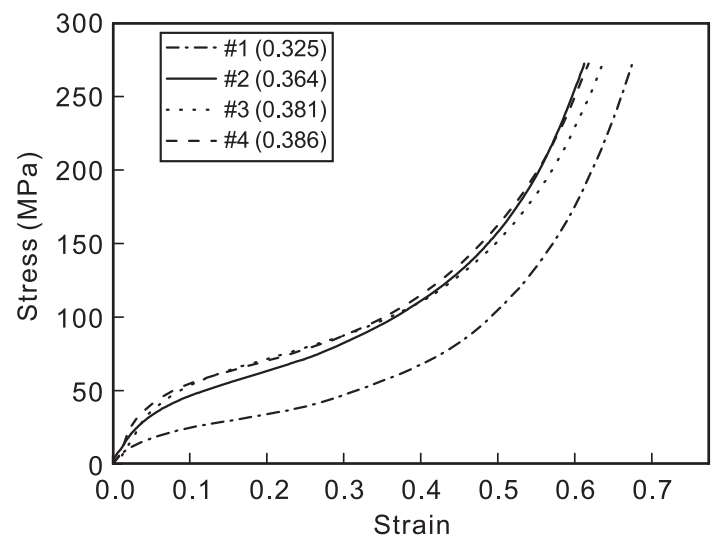

Fig. 7: Quasi-static compressive stress-strain curves of aluminum foam specimens

influence of relative density on the constitutive model is not unitary, and the stress is changed by the coupling effect of the relative density and the strain at that time, which can be represented by power series. Therefore, the relative density effect can be determined by Eq. (8):

$$
G\left(\frac{\rho}{\rho_{0}}, \varepsilon\right)=\left(\frac{\rho}{\rho_{0}}\right)^{A} \cdot e^{-B\left(\frac{\rho_{\mathrm{r}}}{\rho_{0}}-1\right) \varepsilon}
$$

where, $\rho_{0}$ is the selected reference density, which is generally set at the minimum value of $0.325 ; \rho_{\mathrm{r}}$ is the relative density of aluminum foam, $\varepsilon$ is the strain value; $A$ and $B$ are the fitting parameters.

The shape function can reflect the shape and trend of the stress-strain curve of the materials. Aluminum foam material is a kind of nonlinear material with a relatively complex structure, and its nonlinear characteristics are very complicated. Nonlinear materials can be divided into four types: piecewise linear type, cubic function type, tangent type and hyperbolic tangent type ${ }^{[24]}$. Because the stress-strain curve of aluminum foam exhibits a distinct three-stage characteristic, the shape characteristics of the curve cannot be described through a single shape function. Therefore, the quasi-static compressive stressstrain curve for aluminum foam is considered to be defined by the combination of several forms of shape function. According to the shape characteristics of the four commonly used shape functions, combined with the quasi-static compressive stressstrain curve of a typical foam material, it can be seen that the piecewise linear type can reflect the characteristics of the stressstrain curve of the initial elastic stage of the composite material; the hyperbolic tangent type can reflect the curve characteristics with the stress slowly increasing as the strain rises during the material's yielding platform stage; and the tangent type can reflect the curve characteristics with the sharp stress increase during the material compaction stage. Therefore, the shape function of the aluminum foam is established as Eq. (9):

$$
f(\varepsilon)=k_{1} \varepsilon+k_{2} \tan h\left(k_{3} \varepsilon\right)+k_{4} \tan h\left(k_{5} \varepsilon\right)
$$

In conclusion, considering the effect of relative density and shape function, the final form of the quasi-static constitutive model of a SOAF is given as Eq. (10):

$$
\begin{aligned}
\sigma= & {\left[\left(\frac{\rho}{\rho_{0}}\right)^{A} \cdot e^{-B\left(\frac{\rho_{r}}{\rho_{0}}-1\right) \varepsilon}\right] \cdot\left[k_{1} \varepsilon+k_{2} \tanh \left(k_{3} \varepsilon\right)\right.} \\
& \left.+k_{4} \tan \left(k_{5} \varepsilon\right)\right]
\end{aligned}
$$

This constitutive model is suitable for elastoplastic foamed metal materials under quasi-static compression conditions without considering the effects of strain rate and temperature. Because the cells of the foamed material in the compaction stage are completely compacted, and the force-receiving area of the material at this time is already different from the original crosssectional area, the characteristics reflected by the stress-strain curve of the material are not the characteristics of the foam material. Thus, the established constitutive model only studies the stress-strain response of the elastic phase and the plastic deformation phase.

\subsection{Parameter fitting for constitutive model}

The parameter fitting is a process in which the unknown parameters in a model are calculated by building up a mathematical model and then simulating the model's laws, assuming discrete test or real data points are known. During the parameter fitting, the most important thing is to describe the expression of the mathematical model.

(1) Parameter fitting affected by shape function

In MATLAB, the parameters $k_{1}, k_{2}, k_{3}, k_{4}$, and $k_{5}$ in Eq. (9) are identified by the cftool fitting tool of MATLAB and by using stress and strain test data of the specimens. Selecting the relative density 0.325 as the reference relative density, based on the stress-strain data of the quasi-static compression test specimen, the five parameters affected by the shape function are obtained by fitting. Taking the influence parameters of the shape function obtained by \#1 standard test piece fitting as reference, which are shown in Table 1, the fitting result is indicated by the correlation coefficient $R$-square, which is 0.9999 , indicating that the fitting result is very good. The fitting result curve is shown in Fig. 8 (a).

(2) Parameter fitting affected by relative density

The reference relative density is still selected as 0.325 , and the five shape function influence parameters obtained by the fitting are entered into the original constitutive model, then the fitting of the constitutive equation is given for the $\# 2, \# 3$, and \#4 aluminum foam specimens with the relative densities of

Table 1: Parameters affected by shape function of \#1 specimen

\begin{tabular}{ccccccc} 
Specimen & $\boldsymbol{k}_{1}$ & $\boldsymbol{k}_{2}$ & $\boldsymbol{k}_{3}$ & $\boldsymbol{k}_{4}$ & $\boldsymbol{k}_{5}$ & $\boldsymbol{R}$-square \\
\hline$\# 1$ & -256.4 & 19.49 & 21.18 & 181.4 & 1.734 & 0.9999
\end{tabular}



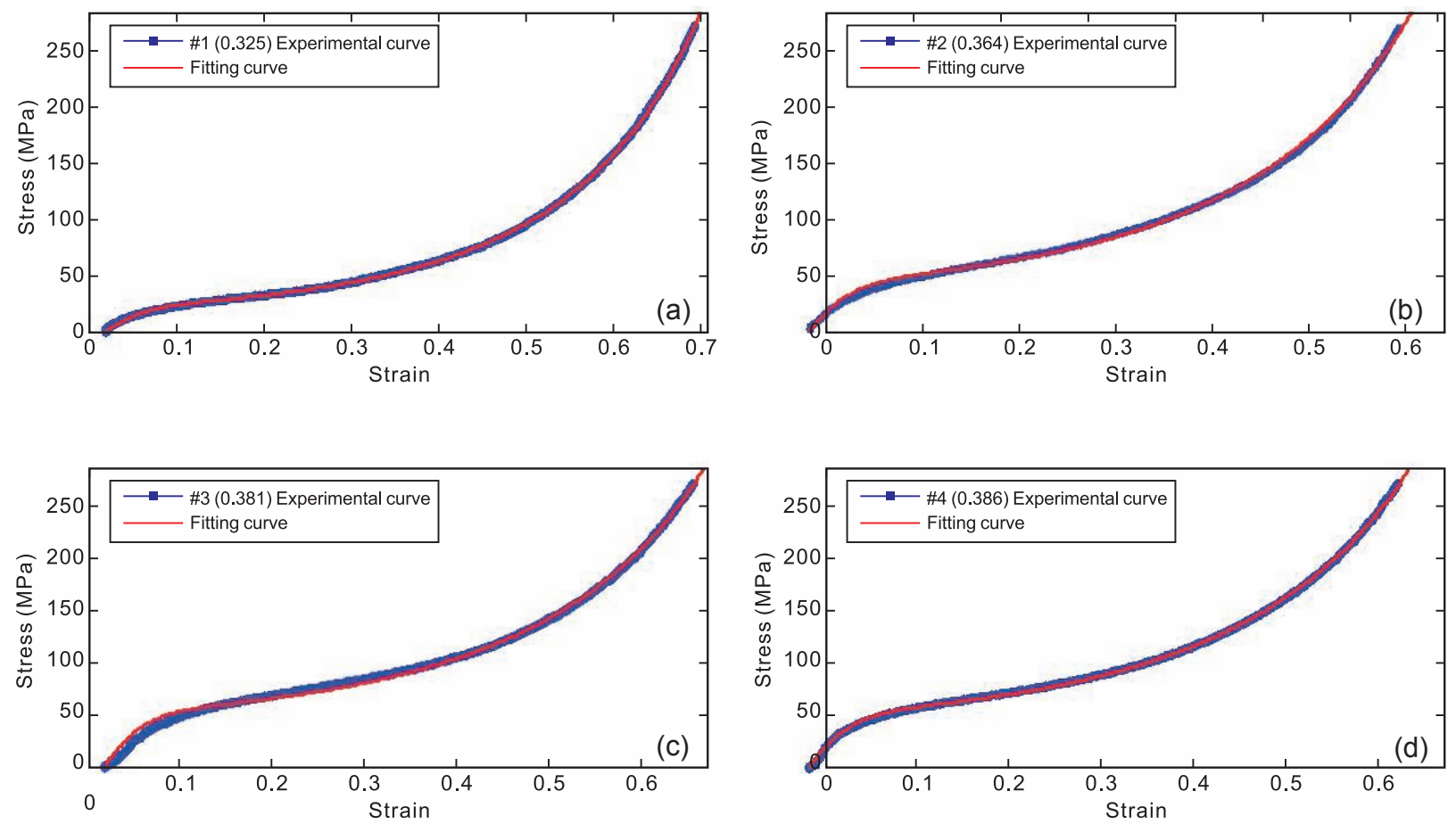

Fig. 8: Fitting curve and test curve for constitutive equation of specimens with different relative densities

$0.364,0.381$, and 0.386 , respectively. By using the stress and strain test data of the above mentioned three test specimens, and adopting the cftool fitting tool to identify parameters $A$ and $B$ which are affected by relative density in Eq. (10), the parameters affected by the relative density of each test specimen are given in Table 2. The comparison results of the fitting result curve and the experimental curve are shown in Fig. 8 (b-d).

Table 2: Parameters affected by relative density

\begin{tabular}{cccc|} 
Specimen & $\boldsymbol{A}$ & $\boldsymbol{B}$ & $\boldsymbol{R}$-square \\
\hline \#2 & 6.412 & 5.066 & 0.9989 \\
$\# 3$ & 5.802 & 6.333 & 0.9969 \\
$\# 4$ & 5.199 & 4.834 & 0.9996 \\
\hline
\end{tabular}

\section{Quasi-static compression numerical simulation}

When introducing the established cylindrical SOAF model into ABAQUS/CAE, using square discrete rigid plates with dimensions of $60 \mathrm{~mm} \times 60 \mathrm{~mm}$ to load the upper and lower surfaces of the model, as well as setting a reference point at the center of the two rigid plates, which are named Upper and Lower, respectively, the movement of the reference point can represent the movement of the rigid body. The finite element model of SOAF is shown in Fig. 9.

In this study, the ABAQUS/Standard analysis module is used to solve the quasi-static compression process of the SOAF. The material properties in ABAQUS are specific to the crosssectional features of the material properties. By defining

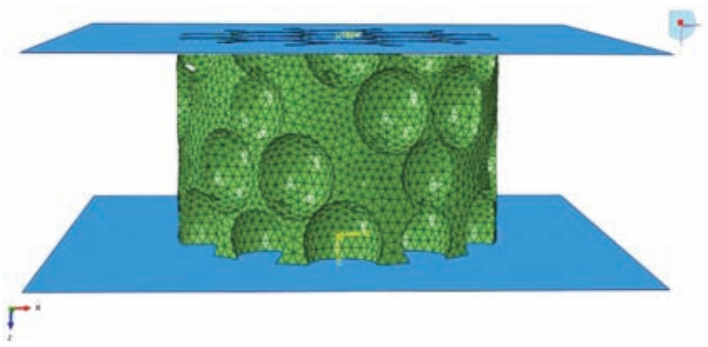

Fig. 9: Quasi-static compression finite element model of SOAF

reasonable materials and cross-section parameters, the materials can exhibit the properties that are very close to the actual mechanical properties. Since the foam material exhibits the characteristics of dense metal in the compact stage, only the material mechanical properties of the linear elastic phase and the plastic deformation phase were studied during the simulation. It can be seen from the quasi-static compression test that the yield limit and the densification strain of the material are 0.04 and 0.45 , respectively. The bulk density, elastic modulus, Poisson's ratio, as well as the yield stress and plastic strain of the plastic phase need to be defined for the material properties in ABAQUS. For the definition of plasticity, the true stress and true strain of the material must be used in ABAQUS, however the nominal stress and nominal strain data of the material are obtained in the quasistatic compression test, therefore the transformation relationship between nominal stress-strain and true stress-strain must be utilized to process the test data. The material property definition of the SOAF is derived from the established constitutive model. The calculation process of the material properties is as follows.

Through the constitutive equation of each test piece, the nominal stress values of 10 points with nominal strains of 0.04 , $0.05,0.10,0.15,0.20,0.25,0.30,0.35,0.40$ and 0.45 were 
obtained, and the elasticity modulus of each test piece was calculated. The selected strain value and the obtained stress value are converted into the real stress and true strain by a conversion relationship between them, and the plastic strain of the material is calculated:

$$
\begin{gathered}
\sigma=\sigma_{\mathrm{n}}\left(1+\varepsilon_{\mathrm{n}}\right) \\
\varepsilon=\ln \left(1+\varepsilon_{\mathrm{n}}\right) \\
\varepsilon^{p l}=\varepsilon-\varepsilon^{e l}=\varepsilon-\frac{\sigma}{E}
\end{gathered}
$$

where $\sigma_{\mathrm{n}}$ is the nominal stress, $\varepsilon_{\mathrm{n}}$ is the nominal strain, $\sigma$ is the
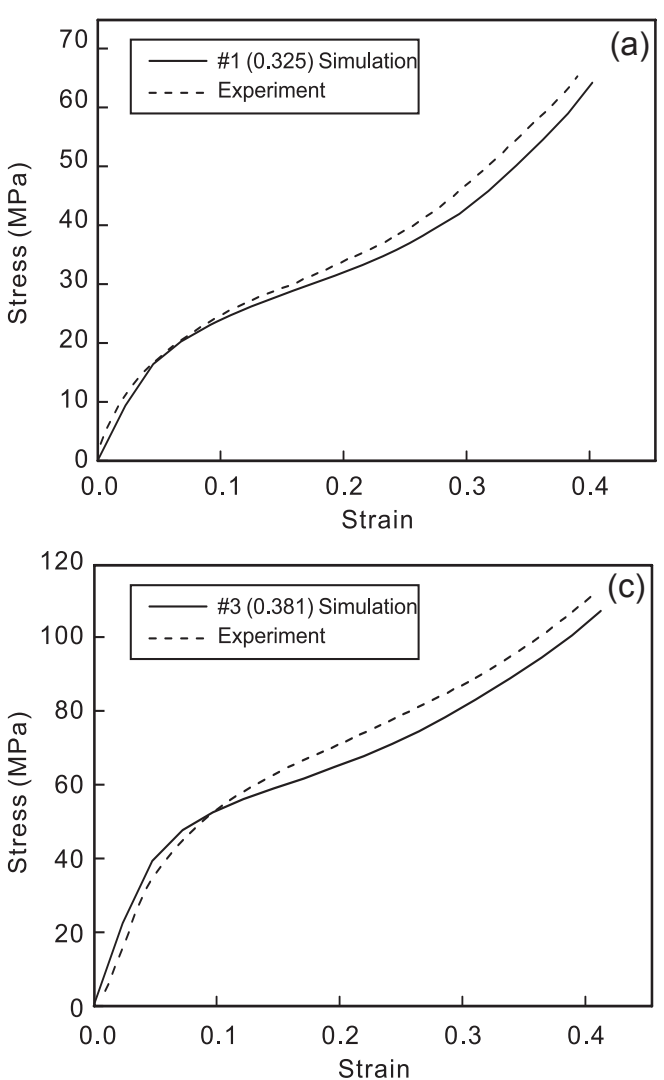

true stress, $\varepsilon$ is the true strain, $\varepsilon^{p l}$ is plastic strain, $\varepsilon^{e l}$ is elastic strain, $E$ is the elastic modulus, and $\varepsilon_{\mathrm{n}}$ is negative values for compression tests. When defining the plastic properties of a material in ABAQUS, the yield stress term is the true stress of the material, and the plastic strain term is filled in with the calculated plastic strain value which on the yield point is zero. Considering the complex cellular structure of the SOAF, the contact between the pore walls will be complex and the contact state will continue to change when subjected to compression load. Therefore, the general contact algorithm is used to define the inner contact of aluminum foam and the contact between the aluminum foam and the upper and lower pressure plates.
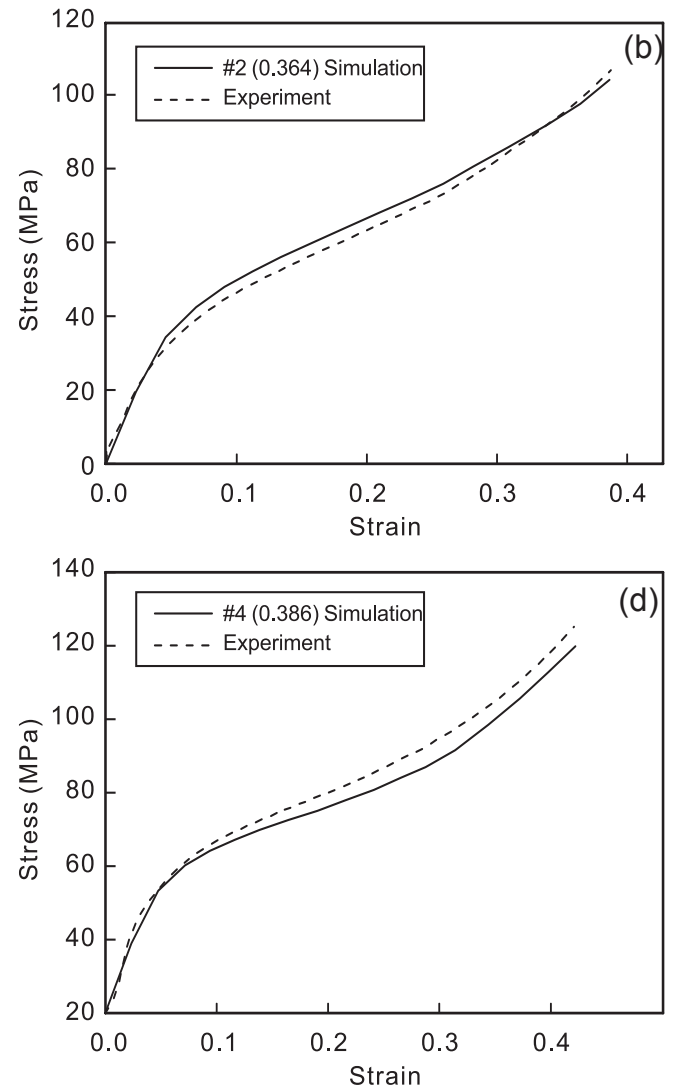

Fig. 10: Simulated and experimental quasi-static compressive stress-strain curves of samples with different relative densities

The quasi-static compressive stress-strain curves for each specimen are shown in Fig. 10.

As seen from Fig. 10, the simulated stress-strain curve of SOAF has the same trend as the one obtained by quasi-static compression experiments, which can verify the feasibility and effectiveness of the established constitutive model and the SOAF 3D model. However, there exist numerical deviations, probably caused by the following reasons: Firstly, because the cellular pore's size and distribution of the real SOAF are irregular but the ones of the simulated model are regular, it is hard to achieve the entire uniformity with the structure of the real material. Secondly, there are defects, such as holes and cracks, in the walls of pores of the real SOAF, which can affect the compression mechanical properties of the materials to a certain extent, while the walls of pores of the simulation model are smooth without defects. Thirdly, the simulation model needs to define the real contact area between the rigid body plate and the surface of the specimen, the calculation of which can probably cause deviation because of the structural differences between the real specimen and the simulated specimen.

\section{Conclusions}

(1) An algorithm, named spherical core stratification algorithm, for the three-dimensional modeling of spherical aluminum foam was put forward. By this algorithm, a threedimensional model of porous aluminum foam with random pore size and adjustable number of layers was successfully constructed. In order to verify the feasibility of the threedimensional modeling method of spherical open cell aluminum 
foam based on the spherical core stratification algorithm, the spherical core stratification algorithm was used to generate the cylindrical sphere open cell aluminum foam with the size of $\Phi 35 \mathrm{~mm} \times 20 \mathrm{~mm}$ and a pore diameter of $5 \mathrm{~mm}$.

(2) By improving the Sherwood-Frost constitutive model, a quasi-static constitutive equation has been established by taking the impacts on foam materials properties from the relative density and shape functions into consideration, and then all the parameters of the constitutive model were obtained by fitting the static compression experimental data with MATLAB. The fitting results show that the established constitutive equation has a good fitting with the experiment, with a fitting correlation coefficient above 0.99 .

(3) Using the ABAQUS finite element analysis software and the established quasi-static compression constitutive equation of the material, the quasi-static numerical simulation of SOAF was performed. The simulation results indicate that the simulated stress-strain curve had the same trend with the one obtained by the quasi-static compression experiment, but with a small deviation due to the difference between the simulation model and real aluminum foam structure. Therefore, the algorithm should be optimized in this aspect in subsequent studies.

\section{References}

[1] Ji H K, Kim D, Lee M G, et al. Multiscale Analysis of OpenCell Aluminum Foam for Impact Energy Absorption. Journal of Materials Engineering \& Performance, 2016, 25(9): 1-8.

[2] Shi T, Chen X, Cheng Y, et al. Foaming Process and Properties of 6063 Aluminum Foams by Melt Foaming Method. Materials Transactions, 2017,58(2): 243-248.

[3] Wang Z J, Zhou J, Wang J, et al. A novel Fault Diagnosis Method of Gearbox Based on Maximum Kurtosis Spectral Entropy Deconvolution. IEEE Access, 2019, 7(1): 29520-29532.

[4] Wang Z J, Wang J Y, Du W H. Research and Application of Improved Adaptive MOMEDA Fault Diagnosis Method. Measurement, 2019, Doi: 10.1016/j.measurement.2019.03.033.

[5] Wang Z J, Wang J Y, Du W H. Research on Fault Diagnosis of Gearbox with Improved Variational Mode Decomposition. Sensors, 2018, 18(10): 1-16.

[6] Wang Z J, He G F, Du W H, et al. Application of Parameter Optimized Variational Mode Decomposition Method in Fault Diagnosis of Gearbox. IEEE Access,Doi: 10.1109/ ACCESS.2019.2909300.

[7] Gibson I J, Ashby M F. The mechanics of three-dimensional cellular materials. Royal Society of London, 1982: 43-59.

[8] Zhang X, Tang L, Liu Z, et al. Yield Properties of Closed-Cell Aluminum Foam under Triaxial Loadings by a 3D Voronoi Model. Mechanics of Materials, 2017, 104: 73-84.

[9] Zhang X, Wang R, Liu J, et al. A numerical method for the ballistic performance prediction of the sandwiched open cell aluminum foam under hypervelocity impact. Aerospace Science \& Technology, 2018, 75: 254-260.
[10] Nie Z, Lin $Y$, Tong Q. Numerical investigation of pressure drop and heat transfer through open cell foams with 3D LaguerreVoronoimodel. International Journal of Heat \& Mass Transfer, 2017, 113: 819-839.

[11] Randrianalisoa J, Baillis D, Martin C L, et al. Microstructure effects on thermal conductivity of open-cell foams generated from the Laguerre-Voronoï tessellation method. International Journal of Thermal Sciences, 2015, 98: 277-286.

[12] Nasrabadi A A M, Hedayati R, Sadighi M. Numerical and experimental study of the mechanical response of aluminum foams under compressive loading using CT data. Journal of Theoretical \& Applied Mechanics, 2016, 54: 1357-1368.

[13] Ranut P, Nobile E, Mancini L. High resolution microtomographybased CFD simulation of flow andheat transfer in aluminum metal foams. Applied Thermal Engineering, 2014, 69(1-2): 230-240.

[14] Zhu X, Ai S, Lu X, et al. Thermal conductivity of closed-cell aluminum foam based on the 3D geometrical reconstruction. International Journal of Heat \& Mass Transfer, 2014, 72(5): 242-249.

[15] Zhu X, Ai S, Lu X, et al. Collapse models of aluminum foam sandwiches under static three-point bending based on 3D geometrical reconstruction. Computational Materials Science, 2014, 85(4): 38-45.

[16] Hangai,Yoshihiko, Yamaguchi, et al. Deformation Behavior Estimation of Aluminum Foam by X-ray CT Image-basedFinite Element Analysis. Metallurgical \& Materials Transactions A, 2013, 44(4): 1880-1886.

[17] Jeon I, Asahina T, Kang K J, et al. Finite element simulation of the plastic collapse of closed-cell aluminum foams with X-ray computed tomography. Mechanics of Materials, 2010, 42(3): 227-236.

[18] Danuta M, Tadeusz N, Roman G. Numerical and experimental aluminum foam microstructure testing with the use of computed tomography. Computational Materials Science, 2012, 64(64): 90-95.

[19] Petit C, Maire E, Meille S, et al. Two-scale study of the fracture of an aluminum foam by X-ray tomography and finite element modeling. Materials \& Design, 2017, 120: 117-127.

[20] Fang H, Bi J, Zhang C, et al. A Constitutive Model of Aluminum Foam for Crash Simulations. International Journal of Non-Linear Mechanics, 2017, 90: 124-136.

[21] Sherwood J A, Frost C C. Constitutive modeling and simulation of energy absorbing polyurethane foam under impact loading. Polymer Engineering \& Science, 2010, 32(16): 1138-1146.

[22] Deshpande V S, Fleck N A. Isotropic constitutive models for metallic foams. Journal of the Mechanics \& Physics of Solids, 2000, 48(6): 1253-1283.

[23] Avalle M, Belingardi G, Ibba A. Mechanical models of cellular solids: Parameters identification from experimental tests. International Journal of Impact Engineering, 2007, 34(1): 3-27.

[24] Hua G, Chao X, Junhui Y, et al. Research on Dynamic Accumulation Effect and Constitutive Model of Aluminum Foams Under Dynamic Impact. International Journal of Metalcasting, 2018,13(1): 146-157. 